\title{
TOPOLOGY OF DEFINABLE ABELIAN GROUPS IN O-MINIMAL STRUCTURES
}

\author{
ELÍAS BARO AND ALESSANDRO BERARDUCCI
}

\begin{abstract}
In this note we prove that every definably connected, definably compact abelian definable group $G$ in an o-minimal expansion of a real closed field with $\operatorname{dim}(G) \neq 4$ is definably homeomorphic to a torus of the same dimension. Moreover, in the semialgebraic case the result holds for all dimensions.
\end{abstract}

\section{INTRODUCTION}

Let $M$ be an o-minimal expansion of a real closed field. Let $H$ be a definable group in $M$ equipped with Pillay's topology in [Pi:88]. So when $M$ is an expansion of the real line $H$ is a real Lie group, and in general it is an " $M$-Lie group". An example of definable group is the $n$-torus $\mathbb{T}^{n}(M)$ over $M$, defined as the poly-interval $[0,1)^{n}$ in $M$ with the sum operation modulo 1 . When $M$ is the real field $\mathbb{R}_{\text {field }}$ this coincides with the classical torus $(\mathbb{R} / \mathbb{Z})^{n}$. Let us recall that $(\mathbb{R} / \mathbb{Z})^{n}$ is the only compact abelian connected Lie group of dimension $n$ up to Lie-isomorphisms.

In the rest of the paper we fix a definably connected, definably compact, definable abelian group $G$ in $M$ of dimension $n$, endowed with Pillay's topology. There are three natural questions:

(1) Is $G$ definably isomorphic to the $n$-torus $\mathbb{T}^{n}(M)$ ?

(2) Is $G$ definably homeomorphic to $\mathbb{T}^{n}(M)$ ?

(3) Is $G$ definably homotopy equivalent to $\mathbb{T}^{n}(M)$ ?

The answer to question (1) is clearly no. For instance $[0,1) \subset \mathbb{R}$ modulo 1 is Lie isomorphic to $S O(2, \mathbb{R})$ but the isomorphism is not semialgebraic (note, however, that they are semialgebraically homeomorphic). Instead, we could ask if $G$ is definably isomorphic to a product of 1-dimensional definable subgroups. But it turns out that this question still has a negative answer even for $M=\mathbb{R}_{\text {field }}$. Indeed it is possible that $\operatorname{dim}(G)>1$, but $G$ has no subgroups of dimension one definable in $\mathbb{R}_{\text {field }}[$ PeSte:99, Example 5.2].

On the other hand the homotopy problem (3) has a positive answer by [BeMaOt:08, Theorem 3.4].

In this note we deal with the homeomorphism problem (2), giving a positive solution when $\operatorname{dim}(G) \neq 4$ (see Theorem 3.1). We also show that when $G$ is semialgebraic, namely $M$ is a real closed field without additional structure, then

Date: Feb. 11, 2011.

2010 Mathematics Subject Classification. 03C64.

Key words and phrases. o-Minimality, Definable Groups.

The first author is partially supported by MTM2008-00272 and Grupos UCM 910444. The second author is partially supported by PRIN 2007PRYAAF 004: O-minimalità - Metodi e modelli non standard - Teoria degli insiemi. 
the result holds in all dimensions (see Theorem 2.1). We point out that the one dimensional case was already proved in [St:94].

The assumption that $G$ is definably compact and definably connected is not very restrictive. In fact by [PeSta:05, Thm.5.1, Thm.5.7] and [PeSte:99, Thm.1.2], the study of the topology of definable abelian groups can be reduced to the definably compact case. So as a corollary we obtain, with a small proviso, that a definably connected abelian n-dimensional group is definably homeomorphic to a space of the form $\mathbb{T}^{m}(M) \times M^{k}$ with $m+k=n$. The proviso is vacuous in the semialgebraic case, while in the general case we require that $m \neq 4$, where $m$ is $n$ minus the dimension of the maximal torsion free definable subgroup of $G$.

Suitable versions of problems (1),(2),(3) can be posed in the non-abelian case. This can be done as follows. Given a definable group $G$, there is a canonical real Lie group $G / G^{00}$ associated to $G$ (by [Pi:04] and [BOPP:05]). By [Ba:10] and [BeMa:10] when $G$ is definably compact and definably connected, the isomorphism type of $G / G^{00}$ determines $G$ up to definable homotopy equivalence. One can ask whether the isomorphism type of $G / G^{00}$ determines $G$ up to definable homeomorphism. The results in [Ma:10] reduce the question to the abelian case, which is the one we consider in this paper. Finally let us observe that by [Co:09t, Thm.3.8.8] (see also [Co:09]) the study of the topology of a definable group reduces to the definably compact case.

We shall make use of the "o-minimal Hauptvermutung" proved by M. Shiota in [Sh:97, Chapter III] when $M$ is an expansion of $\mathbb{R}_{\text {field }}$, and extended in [Sh:10, §2] to the case when $M$ is an o-minimal expansion of an arbitrary real closed field.

Fact 1.1. (o-minimal Hauptvermutung) Let $K$ and $L$ be finite simplicial complexes. Let $M$ be an o-minimal expansion of a real closed field. If there exists a definable, in $M$, homeomorphism from $|K|$ to $|L|$, then there is a $P L$-isomorphism from $|K|$ to $|L|$.

Here $|K|$ denotes a geometrical realization, in $M$, of the simplicial complex $K$. In this note all simplicial complexes are closed and finite, so that $|K|(M)$ is always definably compact. By a "PL map" we always mean "finitely PL map", namely the geometrical realization of a simplicial map between finite subdivisions of the relevant complexes. In Section 2 we prove the semialgebraic case of the homeomorphism problem (2) using the Hauptvermutung in the weak form of [Sh:97]. In Section 3 we assume $\operatorname{dim}(G) \neq 4$ and we reduce the general o-minimal case to the semialgebraic case. In this step we need the strong form of the Hauptvermutung (as in [Sh:10]) and the following fact:

Fact 1.2. (Classification of Homotopy tori) Let $X$ be a closed PL-manifold of dimension $n \neq 4$ homotopy equivalent to the standard torus $\mathbb{T}^{n}(\mathbb{R})$ (considered as a PL-manifold under a standard triangulation). Then there is a finite covering $f: \widetilde{X} \rightarrow X$ such that $\tilde{X}$ is PL-homeomorphic to $\mathbb{T}^{n}(\mathbb{R})$.

When $n \geq 5$ a proof of Fact 1.2 can be found in [HsSi:69, Theorem B] and [Wa:69, Corollary]. (See also [Wa:99, Chapter 15A] for a complete development of homotopy tori.) When $n \leq 3$ it turns out that $X$ is already $P L$-homeomorphic to a standard torus. Indeed, for $\operatorname{dim}(X)=1$ or 2 this is well-known and for $\operatorname{dim}(X)=3$ we can use [KiSi:77, Theorem 5.4, pag 249] together with the positive solution of the three dimensional Poincaré's conjecture. Since our intended readership may not be familiar with the notations in [KiSi:77] we add few lines of explanation. The cited 
theorem tells us that $S^{*}\left(\mathbb{T}^{3}\right)=0$. Unraveling the notations this means that if $M_{1}$ and $M_{2}$ are $P L$-manifolds and $f_{i}: M_{i} \rightarrow \mathbb{T}^{3}$ is a homotopy equivalence for $i=1,2$, then there is a $P L$-homeomorphism $h: M_{1} \rightarrow M_{2}$ such that $f_{2} \circ h$ is homotopic to $f_{1}$. In particular, taking $M_{2}=\mathbb{T}^{3}$ and $f_{2}=i d$, we obtain that any $P L$-manifold homotopy equivalent to $\mathbb{T}^{3}$ is $P L$-homeomorphic to $\mathbb{T}^{3}$. This statement (= Borel's conjecture for $\mathbb{T}^{3}$ ) is known to imply Poincaré's conjecture in dimension $3[\mathrm{~F}: 96$, $\S 1.4]$, which was not known when [KiSi:77] was written. The solution of the riddle lies in a note hidden inside the proof of Theorem 5.3 in [KiSi:77] whose effect is to modify the definition of $S^{*}$ in dimension 3: "in dimension 3 we supplement this definition by supposing that $M_{1}$ is Poincaré, i.e., contains no fake 3-discs". Granted the positive solution to the 3 -dimensional Poincaré's conjecture, the supplement is vacuous.

\section{Semialgebraic CASE}

In this section suppose that $M$ is a real closed field without additional structure. So the definable sets in $M$ coincide with the semialgebraic sets. We prove.

Theorem 2.1. G is semialgebraically homeomorphic to the $n$-standard torus $\mathbb{T}^{n}(M)$.

Proof. By Robson's embedding theorem (see [vdD:98, Theorem 10.1.8]) we can assume that the topology of $G$ (given by [Pi:88]) coincides with the topology induced by the ambient space $M^{n}$. By the triangulation theorem we can then assume that the underlying set $\operatorname{dom}(G)$ is the realization of a $\emptyset$-definable finite simplicial complex $K$. A priori we cannot ensure that the group operation of $G$ is $\emptyset$-definable, but by model completeness of the theory of real closed fields there exist a possibly different group operation on $\operatorname{dom}(G)=|K|$ which is $\emptyset$-definable and continuous with respect to the topology of $|K|$. Since we are only interested in the definable homeomorphism type of $G$ we can assume the group operation is $\emptyset$-definable. We can then consider the group $G(\mathbb{R})$ obtained by interpreting the defining formulas in $\mathbb{R}_{\text {field }}$. By $[\mathrm{Pi}: 88$, Remark 2.6], there is a (unique) Nash group structure on $G(\mathbb{R})$. In particular, $G(\mathbb{R})$ is an abelian compact connected real Lie-group and therefore there is a Lie-isomorphism $f: G(\mathbb{R}) \rightarrow \mathbb{T}^{n}(\mathbb{R})$. We will show that $f$ is definable in some o-minimal expansion of the real field. In fact it is enough to consider the o-minimal structure $\mathbb{R}_{a n}$ studied in [vdD:86]. We need the following:

Fact: Given an analytic function $f$ defined on an open subset $V$ of $\mathbb{R}^{n}$, its restriction to a definable (i.e. semialgebraic) compact subset $K \subset V$ is definable in $\mathbb{R}_{a n}$.

Indeed this is true (almost by definition of $\mathbb{R}_{a n}$ ) when $K$ is a compact polyinterval, and the general case follows by covering $K$ by finitely many poly-intervals contained in $V$. We then obtain:

Claim: The Lie-isomorphism $f: G(\mathbb{R}) \rightarrow \mathbb{T}^{n}(\mathbb{R})$ is definable in $\mathbb{R}_{a n}$.

In fact there are semialgebraic charts making $G(\mathbb{R})$ into a Nash group and for each chart $V, f \mid V$ is analytic. By shrinking the charts we can assume that $f \mid V$ extends to an analytic map on the closure of $V$. So by the above fact $f$ is definable in $\mathbb{R}_{a n}$.

In particular we have proved that there is a homeomorphism $f: G(\mathbb{R}) \rightarrow \mathbb{T}^{n}(\mathbb{R})$ definable in $\mathbb{R}_{a n}$. By the semialgebraic triangulation theorem and the o-minimal Hauptvermutung of [Sh:97], there is a semialgebraic homeomorphism $g: G(\mathbb{R}) \rightarrow$ $\mathbb{T}^{n}(\mathbb{R})$. Moreover, by model completeness of the theory of real closed field, there is 
some $g$ as above which is $\emptyset$-definable in $\mathbb{R}_{\text {field }}$. Interpreting the same formulas in $M$ we obtain a semialgebraic homeomorphism from $G(M)$ to $\mathbb{T}^{n}(M)$ as desired.

\section{General o-minimal Case}

In this section we assume that $M$ is an arbitrary o-minimal expansion of a real closed field. We will prove:

Theorem 3.1. If $n=\operatorname{dim}(G) \neq 4, G$ is definably homeomorphic to the $n$-torus $\mathbb{T}^{n}(M)$.

As above, we can assume that Pillay's topology on $G$ coincides with the topology induced by the ambient space $M^{n}$, and by the triangulation theorem we can then assume that $\operatorname{dom}(G)$ is the geometrical realization $|K|(M)$ of a finite simplicial complex $K$. We need:

Lemma 3.2. If $n=\operatorname{dim}(G) \neq 4, \operatorname{dom}(G)=|K|(M)$ admits a semialgebraic abelian group operation (possibly unrelated to the original one).

Theorem 3.1 follows at once from the Lemma and the semialgebraic case (Theorem 2.1). So it remains to prove the lemma.

Proof of Lemma 3.2. Note that $\operatorname{dom}(G)=|K|(M)$ is at the same time a closed definable manifold (with Pillay's topology) and the realization, over $M$, of a finite simplicial complex. By Shiota's o-minimal Hauptvermutung in [Sh:10], it easily follows (see Fact 3.3 below) that $|K|(M)$ is a closed $P L$-manifold "over $M$ ". This is equivalent to say that the closed star of each vertex of $K$ is $P L$-homeomorphic to the standard simplex of the correct dimension. By model completeness of the theory of real closed fields, the same holds over $\mathbb{R}$. Namely $|K|(\mathbb{R})$ is a $P L$-manifold (but we have no way of inheriting the definable group structure of $|K|(M))$. Moreover $|K|(\mathbb{R})$ is homotopy equivalent to the standard torus $\mathbb{T}^{n}(\mathbb{R})$. Indeed, by [BeMaOt:08, Theorem 3.4] there exists a definable homotopy equivalence from $\operatorname{dom}(G)=|K|(M)$ to $\mathbb{T}^{n}(M)$ and therefore by [BaOt:10, Theorem 3.1] there is a semialgebraic homotopy equivalence from $|K|(\mathbb{R})$ to $\mathbb{T}^{n}(\mathbb{R})$. Since $n=\operatorname{dim}(G) \neq 4$, by Fact $1.2,|K|(\mathbb{R})$ has a finite $P L$-cover which is $P L$-homeomorphic to $\mathbb{T}^{n}(\mathbb{R})$. Namely we have a PL-covering $f: \mathbb{T}^{n}(\mathbb{R}) \rightarrow|K|(\mathbb{R})$ with finite fibers. By model completeness we can assume that $f$ is defined without parameters. So we can go back to $M$ and get a semialgebraic (actually $P L$ ) covering

$$
f: \mathbb{T}(M)^{n} \rightarrow|K|(M)=\operatorname{dom}(G) .
$$

But on $\operatorname{dom}(G)$ we have a definable group operation that can be lifted to $\mathbb{T}^{n}(M)$ via $f$ (by uniform lifting of paths). So we get a definable group operation $*$ on $\mathbb{T}^{n}(M)$ making $f$ into a definable covering homomorphism with a finite kernel $\Gamma<\left(\mathbb{T}^{n}(M), *\right)$. Note that $*$ may not coincide with the natural group operation on $\mathbb{T}^{n}(M)$ (the sum mod 1), so in particular it need not be semialgebraic. In any case however $\left(\mathbb{T}^{n}(M), *\right)$ is an abelian group. Therefore there is $k$ such that $\Gamma$ is contained in the $k$-torsion subgroup $\left(\mathbb{T}^{n}(M), *\right)[k]$ of $\left(\mathbb{T}^{n}(M), *\right)$. Our next goal is to obtain a definable group homomorphism

$$
h: G \rightarrow\left(\mathbb{T}(M)^{n}, *\right) .
$$

Write for simplicity $\mathbb{T}^{n}$ for $\mathbb{T}^{n}(M)$. Now $G$ is definably isomorphic to $\left(\mathbb{T}^{n}, *\right) / \Gamma$ and since $\Gamma<\left(\mathbb{T}^{n}, *\right)[k]$ there is a definable covering from $\left(\mathbb{T}^{n}, *\right) / \Gamma$ to $\left(\mathbb{T}^{n}, *\right) /\left(\mathbb{T}^{n}, *\right)[k]$. 
The latter group is definably isomorphic to $\left(\mathbb{T}^{n}, *\right)$ because the $k$-torsion subgroup of a definably compact definably connected abelian group $H$ is the kernel of the surjective homomorphism $H \rightarrow H$ sending $x$ to $k x$ (we need the fact that such groups are divisible, as proved in [EdOt:04, Theorem 2.1]). Composing we obtain a finite definable covering homomorphism $h: G \rightarrow\left(\mathbb{T}^{n}, *\right)$. As already remarked * may not be semialgebraic. However on $\mathbb{T}^{n}$ we also have a semialgebraic group operation - (the sum mod 1). The idea is to use the covering map $h$ (seen just as a continuous map, not as a group homomorphism) to lift the semialgebraic group operation - to a semialgebraic group operation on $\operatorname{dom}(G)$. The problem however is that $h$ is not semialgebraic. However by [EdJoPe:10, Corollary 2.2], each definable cover of a semialgebraic group, is equivalent to a semialgebraic cover. So there is a semialgebraic covering homomorphism $h^{\prime}: G^{\prime} \rightarrow\left(\mathbb{T}^{n}, \cdot\right)$ and a definable homeomorphism $\psi: \operatorname{dom}(G) \rightarrow \operatorname{dom}\left(G^{\prime}\right)$ commuting with $h$ and $h^{\prime}$. But $\operatorname{dom}(G)$ and $\operatorname{dom}\left(G^{\prime}\right)$ are semialgebraic, so by the Hauptvermutung (combined with the triangulation theorem) there is a semialgebraic homeomorphism $\phi: \operatorname{dom}(G) \rightarrow$ $\operatorname{dom}\left(G^{\prime}\right)$. Now take $h^{\prime} \circ \phi$. This is a semialgebraic covering from $\operatorname{dom}(G)$ to $\left(\mathbb{T}^{n}, \cdot\right)$, and it can be used to lift · to a semialgebraic group operation on $\operatorname{dom}(G)$.

Let us prove the missing fact needed in the above proof.

Fact 3.3. Let $K$ be a finite simplicial complex such that $|K|$ is an $n$-dimensional closed definable manifold. Then $|K|$ is a PL-manifold, namely the star of each vertex of $K$ is $P L$-homeomorphic to the standard n-simplex.

Proof. In this proof simplicial complexes are assumed to be finite but not necessarily closed. We will use without mention the well-known invariance of stars in piecewise linear topology, i.e., the star of a vertex of a closed simplicial complex is PL-isomorphic to the star of that vertex in any simplicial subdivision. Let $\left\{\left(U_{1}, f_{1}\right), \ldots,\left(U_{s}, f_{s}\right)\right\}$ be a definable atlas of $|K|$. That is, each $U_{i}$ is a definable open subset of $|K|$, each $f_{i}$ is a definable homeomorphism from $U_{i}$ to a definable open subset $V_{i}$ of $M^{n}$ (with the usual property on transition maps) and $|K|=\bigcup_{i=1}^{s} U_{i}$. By shrinking of coverings, we can find definable open subsets $W_{1}, \ldots, W_{s}$ of $|K|$ such that $|K|=\bigcup_{i=1}^{s} W_{i}$ and $W_{i} \subset \bar{W}_{i} \subset U_{i}$ for each $i=1, \ldots, s$. Moreover, by the triangulation theorem we can assume that each $V_{i}$ is the realization of an open finite simplicial complex and $f_{i}\left(\overline{W_{i}}\right)$ the realization of a subcomplex. Considering a barycentric subdivision if necessary, we can also assume that the star in $V_{i}$ of each vertex of $f_{i}\left(\overline{W_{i}}\right)$ is a closed finite subcomplex. In particular, since $V_{i}$ is an open subset of $M^{n}$, it follows that the star of each vertex in $f_{i}\left(\overline{W_{i}}\right)$ is PL-isomorphic to a standard $n$-simplex.

Now, again by the triangulation theorem, there exist a definable homeomorphism $\psi:|L| \rightarrow|K|$ compatible with the definable sets $U_{i}, W_{i}$ and $\overline{W_{i}}$. Since $\psi^{-1}\left(\overline{W_{i}}\right)$ and $f_{i}\left(\overline{W_{i}}\right)$ are definable homeomorphic, by the o-minimal Hauptvermutung they are PL-isomorphic. Now, given a vertex $v$ of $L$, the star of $v$ in $L$ is contained in some $\overline{W_{i}}$ and therefore is PL-isomorphic to the star of some vertex of $f_{i}\left(\overline{W_{i}}\right)$. Hence, the star of each vertex of $L$ is PL-isomorphic to a standard $n$-simplex.

By the o-minimal Hauptvermutung, there exist a PL-isomorphism of $|L|$ and $|K|$. Hence we deduce that the star of each vertex of $K$ is PL-isomorphic to the star of a vertex of $L$, which in turn is PL-isomorphic to a standard $n$-simplex.

We have thus completed the proof of the Lemma, and Theorem 3.1 follows. 
A possible attempt to deal with the case $\operatorname{dim}(G)=4$ is to replace $G$ with $G \times \mathbb{T}^{1}$. So by Theorem 3.1 there is definable homeomorphism from $G \times \mathbb{T}^{1}$ to $\mathbb{T}^{5}$. However we do not know whether this implies that there is a definable homeomorphism from $G$ to $\mathbb{T}^{4}$. Finally let us observe that, even in dimension 4, we can always assume $\operatorname{dom}(G)=|K|(M)$ (after a triangulation) and conclude that $|K|(\mathbb{R})$ is homotopy equivalent to $\mathbb{T}^{4}(\mathbb{R})$ (reasoning as in the proof of Lemma 3.2). Moreover by [FrQu:90, §11.5] a fourth dimensional $P L$-manifold homotopy equivalent to a standard torus is homeomorphic to it (although not necessarily $P L$-homeomorphic). So in any case we conclude that $|K|(\mathbb{R})$ is homeomorphic to $\mathbb{T}^{4}(\mathbb{R})$, but since a priori the homeomorphism could be quite wild, there is no obvious way to obtain from these data a definable homeomorphism from $|K|(M)$ to $\mathbb{T}^{4}(M)$.

\section{ACKNOWLEDGEMENTS}

We thank M. Mamino for many discussions regarding the topics of this paper, and R. Benedetti, R. Frigerio, A. Ranicki, M. Shiota and C.T.C. Wall for their comments concerning low dimensional topology and classification of manifolds. This work was done while the first author was visiting the Department of Mathematics of the University of Pisa in the period 6 January - 27 July 2010.

\section{REFERENCES}

[Ba:10] E. Baro, On the o-minimal LS-category, to appear in Israel J. Math.

[BaOt:10] E. Baro and M. Otero, On o-minimal homotopy, Quart. J. Math. 61 (3) (2010), 275-289.

[BeMa:10] A. Berarducci and M. Mamino, On the homotopy type of definable groups in an ominimal structure, to appear in J. London Math. Soc.

[BeMaOt:08] A. Berarducci, M. Mamino and M. Otero, Higher homotopy of groups definable in o-minimal structures, Israel J. Math. 180 (2010), 143-161.

[BOPP:05] A. Berarducci, M. Otero, Y. Peterzil and A. Pillay, A descending chain condition for groups definable in o-minimal structures, Ann. Pure Appl. Logic 134 (2005), 303-313.

[Co:09t] A. Conversano, On the connections between definable groups in o-minimal structures and real Lie groups: the non-compact case, PhD. Thesis, University of Siena, 2009.

[Co:09] A. Conversano, Lie-like decompositions of groups definable in o-minimal structures, 2009, arXiv:0912.4753.

[vdD:86] L. van den Dries, A generalization of the Tarski-Seidenberg theorem, and some nondefinability results, Bull. AMS 15 (1986), 189-193.

[vdD:98] L. van den Dries, Tame topology and o-minimal structures, London Mathematical Society Lecture Note Series, 248, Cambridge University Press, 1998.

[EdJoPe:10] M. Edmundo, G. Jones and N. Peatfield, Invariance results for definable extensions of groups, Arch. Math. Logic, DOI: 10.1007/s00153-010-0196-5, 2010.

[EdOt:04] M. Edmundo and M. Otero, Definably compact abelian groups, J. Math. Logic 4 (2) (2004), 163-180.

[F:96] F.T. Farrell, Lectures on surgical methods in rigidity, Tata Institute of Fundamental Research, Springer-Verlag, Berlin, 1996.

[FrQu:90] M.H. Freedman and F. Quinn, Topology of 4-manifolds, Princeton Mathematical Series (39), Princeton University Press, Princeton, NJ, 1990.

[HsSi:69] W.C. Hsiang and J.L. Shaneson, Fake tori, the annulus conjecture, and the conjectures of Kirby, Proc. Nat. Acad. Sci. U.S.A. 62 (1969), 687-691.

[KiSi:77] R.C. Kirby and L.C. Siebenmann, Foundational essays on topological manifolds, smoothings, and triangulations, Annals of Mathematics Studies (88), Princeton University Press, Princeton, N.J., University of Tokyo Press, Tokyo, 1977.

[Ma:10] M. Mamino, Splitting definably compact groups in o-minimal structures, 2010, arXiv:1001.2229.

[PeSta:05] Y. Peterzil and S. Starchenko, On torsion-free groups in o-minimal structures, Illinois J. Math., 49 (4) (2005), pp. 1299-1321. 
[PeSte:99] Y. Peterzil and C. Steinhorn, Definable compactness and definable subgroups of ominimal groups, J. London Math. Soc. 59 (2) (1999), 769-786.

[Pi:88] A. Pillay, On groups and fields definable in o-minimal structures, Journal of Pure and Applied Algebra, vol. 53 (3) (1988), 239-255.

[Pi:04] A. Pillay, Type-definability, compact Lie groups, and o-minimality, J. Math. Logic 4 (2004), $147-162$.

[Sh:97] M. Shiota, Geometry of subanalytic and semialgebraic sets, Progress in Mathematics, 150, Birkhuser Boston, Inc., Boston, MA, 1997.

[Sh:10] M. Shiota, PL and differential topology in o-minimal structures, 2010, arXiv:1002.1508.

[St:94] A.W. Strzebonski, One dimensional groups definable in o-minimal structures, J. Pure Appl. Algebra 96 (2) (1994), 203-214.

[Wa:69] C.T.C. Wall, On the homotopy tori and the annulus theorem, Bull. London Math. Soc. 1 (1969), 95-97.

[Wa:99] C.T.C. Wall, Surgery on compact manifolds, Mathematical Surveys and Monographs (69), American Mathematical Society, Providence, RI, 1999.

Departamento de Álgebra, Facultad de Matemáticas, Universidad Complutense de MADRID, 28040 MADRID

E-mail address: eliasbaro@pdi.ucm.es

Università di Pisa, Dipartimento di Matematica, Largo Bruno Pontecorvo 5, 56127 Pisa, Italy

E-mail address: berardu@dm.unipi.it 